\title{
Per la fonte della Sequenza volgare di Santa Eulalia.
}

È risaputo che il racconto del martirio di Sant'Eulalia, quale ci si offre nella sequenza francese, diverge tanto dalle altre narrazioni, che dello stesso martirio sono giunte fino a noi (Diez, Altrom. Sprachdenk. I5, Koschwitz, Commentar 55 sgg.), che si sarebbe quasi tentati di credere ad una Eulalia diversa dalla spagnuola. ${ }^{1}$ Parecchi e validi argomenti devono però distoglierci dal cedere alla tentazione: così il fatto che di Eulalie veramente celebri non vi ha che la iberica ${ }^{2}$, l'accordo nel far volare al cielo la santa in forma

1 Dico 'spagnuola' poichè vita, morte e miracoli delle due Eulalie iberiche, la barcellonese ciod e la lusitana, si confondono talmente da rendere legittimo il dubbio che le due eroine sieno in origine una sola e stessa persona; cfr. España sagrada XXIX 287 sgg., 302 sgg., AA. SS. Febr. II 576.

2 C'è, o almeno pare ci sia, un' Eulalia romana di cui tocca il Diez (o. c. 16) riferendosi però alla España sagrada XXIX 305, dove se ne ragiona con queste parole: "Algunos martirologios ponen una Santa Eulalia en Roma sobre el dia II de diciembre, como se vé en Florentinio; y si ésta murio degollada, pudo equivocarse con la Española el genero de la muerte." Il Florentinio (Vetustius occidentalis ecclesiae martyrologium; Lucca 1668) poi, dopo citato, a pp. 1025-26, un martirologio nel quale compare un' Eulalia martirizzata a Roma, soggiunge: "Eulaliam inter Romanas martyres numeratam alibi non invenio. Nisi cuius reliquias a coemeterio, sive Callixti, sive Priscillae extractas nostro saeculo, et Antverpiam allatas ibidem venerationi expositas narrat Bollandus in Actis SS. ad diem 28. Februarii." E gli AA. SS. parlano infatti di questi sacri ossami, fra i quali si trovavano de' frantumi di cranio di una Eulalia vergine e martire, trasportati da Roma ad Anversa a meta circa il sec. XVII. Ma chi poi fossero questa Eulalia e i suoi compagni gli AA. SS. non ce lo sanno dire: "De ipsis porro Martyribus nihil nobis compertum, nisi ex coemeteriis Romanis, quae diximus, extractas eorum reliquias: qua vixerint aetate, quibus suppliciis fidem testati, sub quibus tyrannis, plane ignoratur. Sunt quidem in Martyrologiis nonnulli Martyres iisdem nominibus, quibus et hi, insigniti; verum eosdem esse, non possumus nisi temere pronuntiarc, neque universim omnes esse diversos"; e più oltre: "nulla Romae Eulalia adhuc nobis reperta".

Un' altra Eulalia allega dai Martirologii il Florentinio, pp. $3^{n 9}-3^{10}$, accompagnando però la citazione con queste parole: "An vero, quae postremo recensetur, Eulalia Hispana martyr Barcinonensis sit, suspicari ex eo licet, quod hac eadem die 12 Februarii aliqua martyrologia eandem recolant .... Diversam tamen facile credimus, quod, in Italia, et cum Italis aliis, adhuc obscuris martyribus, recolatur die duodecimi Januarii. Ferrarius apud Bollandum Uxenti in Salentinis, Eulaliam virginem et martyrem coli refert, quam Barcinonensem, translatis in Italiam reliquiis, suspicatur. At Ferrarius in $\mathrm{Ca}$ talogo Sanctorum Euleliam vocat, tam in laterculo, quam in notationibus. In 
di colomba, e infine e sopratutto il rapporto intimo che da una parte lega la sequenza volgare al cantico latino, che nel ms. le precede, e dall'altra questo stesso cantico all'inno di Prudenzio, che si considera come il più autentico degli atti che riguardano la Vergine spagnuola.

Le differenze che corrono tra il canto di Prudenzio e la prosa francese consistono in ciò: a) che questa fa intervenire come giudice, anzichè il pretore, l'imperatore Massimiano stesso; b) che, invece dello spirito, fa volare Eulalia stessa al cielo; c) che, secondo la sequenza, Eulalia vien prima gettata sul rogo, poi, risultata innocua la fiamma, decollata colla spada. Secondo Prudenzio invece, Eulalia, dopo subite altre torture 1, vien posta sul rogo e vi perisce.

Le due prime discordanze non sì può dire che sieno di una grande portata: che la mente finisca collo scordare l'esecutore de' decreti imperiali e solo si ricordi di colui onde i decreti stessi emanano, è cosa assai naturale, poichè è su costui che veramente la responsabilità ricade; e per quant'è del volo di Eulalia al cielo, nulla ci vieta di credere ad una maniera spiccia di esprimere il

alio vero catalogo Sanctorum Italiae Eueliam notat Virginem et Martyrem Uxenti in Salentinis die 12 Januarii, idque indice tantum; ad signatum enim diem nihil invenire licuit". Quanto in queste ultime linee afferma il Florentinio, l'ho dovuto constatare anch'io.

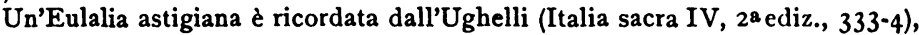
e gli AA. SS., Mart. III 289, così ne toccano: "Passum esse hoc die S. Secundum in urbe Astensi supra diximus, cuius urbis ecclesia dicitur apud Ughellum tomo 4 Italiae sacrae p. 473 et 474 (s'accenna qui alla $1^{2}$ ediz.), inter alios Sanctos Martyres velut primitias, sponso suo Christo obtulisse $\mathrm{S}$. Eulaliam Virginem et Martyrem, cuius corpus ad ecclesiam S. Mariae-Novae deferri mandavisset Julius III Papa anno 1553. Huius alibi non reperimus ullam mentionem, ut vel ideo aliqua iniecta sit suspicio, num forte haec S. Eulalia ex viginti martyrologiis adducta, sit Astae martyrii laurea coronata". - $\mathrm{Ri}$ cordiamo, per ultimo, una Eulalia vergine e martire, 'nulli loco attributa', che si menziona p. 820 di questo stesso vol. degli AA. SS.

Sono dunque queste le Eulalie romane 0 italiane i cui tratti leggendari avrebbero potuto frammischiarsi a quelli dell'Eulalia spagnuola 0 unche sostituirvisi affatto. Ma chi vorrà credere a ciò se tutte sono di una cosi meschina importanza che appena appena se ne conosce il nome? E la loro modestia ha poi maggiore risalto dallo scarso culto che a sante di quel nome, quale poi si sia la loro origine, vien reso in Italia. Così nei due cataloghi del Ferrario (Cat. SS. Italiae in menses duodecim distributus, Milano I6r 3; Cat. gen. SS. qui in Martyrologio romano non sunt, Venezia I625) non è ricordata, a tacere della Ugentina che d'altronde non è Eulalia ma Eulelia, nessuna Eulalia. - Per quant'è dunque di questa Santa, Roma e l'Italia, anzichè regalare ad altri, non avevano che da farsi regalare esse stesse.

1 Prudenzio:

J. . carnifices gemini

Juncea pectora dilacerant

Et latus ungula virgineum

Pulsat utrimque, et ad ossa secat,

Eulalia numerante notas.

Cito secondo l'edizione bodoniana in due volumi (Parma 1788), la migliore che stia a mia disposizione; cfr. vol. I I $43 \mathrm{sgg}$. Altri tormenti e con maggiori dettagli sono narrati dagli altri fonti. 
pensiero, appunto come la madre suol dire, del bambino morto e di cui ha sott'occhio il cadavere, che è andato cogli angioli in paradiso. ${ }^{1}$

Pì̀ grave è invece il dissenso sul terzo punto. Qui, e nelle torture e nell'ordine con cui si succedono, s'ha veramente del nuovo. ${ }^{2}$ Sennonchè le stesse torture, nello stesso ordine e collo stesso esito, si narrano di un'altra eroina cristiana la quale, come più sotto si dirà, già aveva, nelle circostanze generali della sua vita, molto di comune colla nostra. 亡 questa Sant'Agnese. Anche costei fu dannata al fuoco; anche costei, uscita incolume dalla fierissima prova, venne poi decapitata. - Ma la concordanza, che qui risulta evidente, va, a mio vedere, ancora più in là. La vergine romana, prima che per il fuoco e per la spada, aveva dovuto passare per un supplizio ben più duro: era stata rinchiusa in un lupanare, dove solo la grazia divina potè far sì che il di lei fiore rimanesse indelibato (AA. SS. Jan. II 3 '5o sgg.; nel racconto di Prudenzio I 297 sgg., manca il supplizio del fuoco). Ora io credo di ravvisare questo tratto del martirio di Agnese anche nella sequenza volgare di Eulalia, nei versi :

\section{Melz sostendreiet les empedementz ${ }^{3}$ \\ Quelle perdesse sa virginitet. $(16-17)$.}

I versi del componimento che precedono a questi non contengono, è vero, nessuna allusione esplicita ad un attentato contro la pudicizia della vergine, attentato di cui tacciono del resto anche

1 La colomba, del resto, era già di per se come un simbolo dello spirito. - Non mi celo tultavia che il volo al cielo della salma stessa di Eulalia trae conforto dal fatto che la sequenza omette il iniracolo della neve mandata da Dio per coprire le nude e inanimate membra della vergine. bile Beda.

2 Veramente della decapitazione di Eulalia è gid parola nel venera-

8 Che empedementz dica 'torture' risulta gia chiaro dal contesto. Non inutile tuttavia di qui ricordare il rapporto etimologico che corre (secondo il Diez cui è ora contradetto dal Paul Meyer in Romania XVII 421) tra entraver e travailler, e di constatare che l'evoluzione ideologica inversa ci è poi offerta in géner (géner la circulation impedire la circolazione).

4 Nell'atto riprodotto dalla España sagrada (XIII 400; cfr. anche Catalogus cdd. hagiographicorum bibliothecae regiae bruxellensis I 26I) e citato dal Diez, o. c. pag. 28, Eulalia, senza che sia prima parola di offesa alcuna alla sua verginità, così apostrofa il pretore: "Quid persequeris Christianos et niteris perdere Virgines Dei? Dominus me docuit in veritate sua, nec auferes (non auferas, come, per un errore di stampa, hanno il Diez e quindi il Kosch. witz o. c. p. 98) a me castitatem meam, quia non seduces adolescentiam meam." $E$ il pretore, che qui si chiama Calpurnio ed è evidentemente stupito che una tanto tenera fanciulla nutra un si curioso timore, gli risponde: "O infantula, antequam crescas florem aetatis tuae perdere quaeris"? Dai quali passi risulta chiaro che il pretore nulla intende tramare contro la virtù di Eulalia, e che costei parlava, anzichè per se, per le vergini cristiane in genere. - Della cura gelosa che aveva Eulalia del proprio pudore è parola anche nella vita della Santa (si tratta qui della emeritense) che è riassunta nel $6^{\circ}$ vol. della Anamnesis sive commemoratio sanctorum hispanorum di J. Tamayo Salazar (Lione 165I-59). Ma anche qui non le vien usata violenza nessuna: "Cum ad 
Prudenzio e gli Atti 4; eppure mi par evidente che ne' versi citati, nè io saprei come altrimenti dichiararli, si accenni ad un'offesa di tal genere. Forse il poeta, il quale si dirigeva a un pubblico già informato, e d'altronde, costretto com' era dalle necessità speciali del suo ritmo, non poteva dilungarsi troppo, avrà creduto sufficiente il vago e generico accenno al servizio diabolico, che si trova nel $3^{0}$ verso (uoldrent la faire diaule seruir); e l'avrà creduto tanto più in quanto una eccessiva preoccupazione della decenza poteva forse sussurrargli all'orecchio che, in un canto destinato ad edificare i fedeli, su certe cose e su certi nomi, per quanto innocui, era meglio scivolare che insistere.

Ma se la sequenza veramente sa, come io ritengo, di un tentativo contro la castità di Eulalia, questo doveva, assai verosimilmente, corrispondere a quello che ci è raccontato di Agnese. $\mathrm{Ce}$ ne rende quasi convinti l'analogia nelle ulteriori torture delle due eroine.

L'Eulalia della sequenza si sarebbe dunque attribuito l'intero martirio di Agnese. Che ciò potesse avvenire parrà ben naturale a chi, famigliare coi procedimenti soliti della leggenda, consideri le analogie, che indipendentemente da ogni ulteriore contaminazione, gid offriva la vita delle due vergini: ambedue figlie di alti personaggi romani, ambedue fanciulle 'nondum thoris maritalibus habiles', ambedue immolate, nelle persecuzioni de' primi secoli, per la fede di Cristo. ${ }^{2} \mathrm{Ci}$ voleva di più perchè la leggenda attribuisse poi all'una i supplizi dell'altra? Che nella sequenza nostra la confusione avvenisse a favore (o a discapito come la si prende) di Agnese, si spiega forse da ciò, che nella Francia settentrionale ${ }^{3}$,

locum passionis extra urbem pervenisset ipsa se exuens, quaestionariis tradidit. Sane praecinctorium tantummodo sibi, pro pudoris aspectu ad tegimen sui femoris, reservavit" (p. 444); nè è detto in seguito che gli aguzzini avessero la curiosità di vedere più di quello che la Santa s'adattava a mostrare loro.

'Questo verso del cantico d'Eulalia, che s'accompagna nel ms. alla sequenza volgare, è certo una reminiscenza del 'jugali vix habilem thoro' di Prudenzio. - Circa all'età delle due eroine, Agnese vien indicata come tredicenne, e di Eulalia dice Prudenzio: "Tres hiemes quater attigerat".

2 Anche qualche tratto miracoloso è comune alle due fanciulle; così quello delle chiome che ricoprono le nudità verginali di Agnese quand'è spogliata per essere condotta al lupanare (AA. SS.), e di Eulalia quando sta sul rogo (Prudenzio). V'ha tuttavia questa differenza: che per Agnese il miracolo si manifesta nella straordinaria densità dè capelli, e per Eulalia nella fragranza che ne spira. - Il tratto leggendario de' capelli miracolosamente e straordinariamente folti è di parecchie altre sunte; e così sono di tal natura le chiome che ricoprono le nude ma non immacolate carni di S. Maria Egiziaca nel deserto.

8 Il culto di Sant' Eulalia in Francia dev'essere particolarmente diffuso nella regione meridionale; il che si capisce pensando alla vicinanza e all'importanza dell' Eulalia barcellonese. I dieci nomi di communi francesi, che l'Enciclopedia di Ersch e Gruber, la sola fonte ch'io possa in questo momento consultare, fa risalire al nome di Sant'Eulalia, spettano tutti alla regione di lingua d'oc, avendosi come più settentrionali quattro communi della Dordogne, del Cantal e della Correzze. 
la vergine romana, straordinariamente esaltata dalla Chiesa ed elevata a tipo della fanciulla cristiana vergine e martire a un tempo, avrà avuto un culto e una notorietà di gran lunga superiori al culto e alla notorietà di Eulalia. ${ }^{1}$ Ma questa avrà avuto anch'essa un altare $o$ una cappella, e forse più altari e più cappelle. Orbene stando così le cose, era ovvio che delle popolazioni le quali da una parte sapevan d'Agnese e dall'altra veneravano nella loro parrocchia Eulalia, abbiano creduto, nel loro devoto fervore perquesta, di meglio glorificarla attribuendole gran parte dei tratti dell'altra, cioè della più luminosa. Il poeta trovava poi la tradizione fatta e la accoglieva ne' suoi versi.

1 La venerazione cattolica per S. Agnese doveva poi nella Gallia settentrionale avere speciale alimento dalle ossa della Santa, che, tutte $O$ in parte, si credeva e si crede colà di possedere già da tempi antichissimi (AA. SS. Jan. II 335 sgg.).

C. Salvioni. 